\title{
Article
}

\section{The Guinea-Bissau Twin Registry Update: A Platform for Studying Twin Mortality and Metabolic Disease}

\author{
Morten Bjerregaard-Andersen ${ }^{1,2,3,4}$, Gabriel Marciano Gomes ${ }^{1}$, Ditte Egegaard Hennild ${ }^{1,3,5}$, Dorte Møller Jensen ${ }^{6,7}$, \\ Kaare Christensen ${ }^{8}$, Morten Sodemann ${ }^{1,5}$, Henning Beck-Nielsen ${ }^{3,6}$ and Peter Aaby ${ }^{1,2}$ \\ ${ }^{1}$ Bandim Health Project, INDEPTH Network, Bissau, Guinea-Bissau, ${ }^{2}$ Research Center for Vitamins and Vaccines (CVIVA), Statens Serum Institute, Copenhagen, \\ Denmark, ${ }^{3}$ Department of Endocrinology, Odense University Hospital, Odense, Denmark, ${ }^{4}$ Department of Endocrinology, Hospital of Southwest Denmark, \\ Esbjerg, Denmark, ${ }^{5}$ Department of Infectious Diseases, Odense University Hospital, Odense, Denmark, ${ }^{6}$ Steno Diabetes Center Odense, Odense University \\ Hospital, Odense, Denmark, ${ }^{7}$ Department of Gynaecology and Obstetrics, Odense University Hospital, Odense, Denmark and ${ }^{8}$ Department of Public Health, \\ Danish Twin Registry, University of Southern Denmark, Odense, Denmark
}

\begin{abstract}
Sub-Saharan Africa has the highest natural twinning rate in the world. Unfortunately, due to lack of adequate care during pregnancy, labor and postnatally, twin mortality in Sub-Saharan Africa also remains very high. Thus, it has been estimated that one in five twins dies during the childhood years. In spite of this, surprisingly few twin studies have been conducted in the region, making additional epidemiological data much needed. In 2009, we established one of the first twin registries in Sub-Saharan Africa at the Bandim Health Project in Guinea-Bissau. The registry had two main objectives. First, we wanted to describe the twinning rate and mortality patterns among newborn twins, including mortality risk factors and hospitalization patterns. Such studies can help the local clinicians improve twin health by identifying the most vulnerable children. Second, and in light of the rapidly increasing diabetes rates in Africa, we wanted to use the registry to particularly focus on metabolic disorders. Twins are often born with low birth weight, which according to the 'thrifty phenotype hypothesis' could predispose them to metabolic disorders later in life. Yet, no such 'fetal programming' data have previously been available from African twins despite the fact that nutritional patterns and influences from other factors (e.g., infections) could be markedly different here compared to high-income settings. In this article, we summarize the findings and current status of the Guinea-Bissau twin registry.
\end{abstract}

Keywords: Twin registry; Guinea-Bissau; mortality; fetal programming; metabolic disease; diabetes

(Received 10 May 2019; accepted 10 June 2019; First Published online 18 July 2019)

In Sub-Saharan Africa, twins have traditionally retained a special status. In many areas, they have been a sign of good fortune for the family, though in some settings they have been regarded the opposite way (Pison, 1992). Interestingly, Sub-Saharan Africa has the highest natural twinning rate in the world, with a regional average of 20 twin births per 1000 total births (Pison, 1992). However, in some parts of the region, the twinning rate has been noted to be considerably higher, that is, $40-45 / 1000$ in south-west Nigeria. The reason for this high rate remains unknown, though genetic factors are likely important. Other factors influencing the natural twinning rate may include diet, high parity, maternal history of twinning and socio-economic status (Akinboro et al., 2008).

Disconcertingly, twinning in Africa is associated with numerous risks, which are exacerbated by an often weak health care infrastructure. These risks - related to, for example, low birth weight, prematurity, birth difficulties and poor growth - are particularly pronounced during early life. Thus, one pooled analysis of $90 \mathrm{dem}-$ ographic and health surveys across the continent found that one-

Author for correspondence: Morten Bjerregaard-Andersen, Email: mban@dadlnet.dk Cite this article: Bjerregaard-Andersen M, Marciano Gomes G, Egegaard Hennild D, Møller Jensen D, Christensen K, Sodemann M, Beck-Nielsen H, and Aaby P. (2019) The Guinea-Bissau Twin Registry Update: A Platform for Studying Twin Mortality and Metabolic Disease. Twin Research and Human Genetics 22: 554-560, https://doi.org/10.1017/thg.2019.39 fifth of the twins die before the age of 5 years (Monden \& Smits, 2017). This has a huge impact on the overall childhood mortality in the region (Christensen \& Bjerregaard-Andersen, 2017; Monden \& Smits, 2017).

In 2009, we established one of Africa's first formal twin registries at the Bandim Health Project (BHP) in Guinea-Bissau (Bjerregaard-Andersen et al., 2013a). Prior epidemiological data from our setting had shown high twin mortality (Aaby et al., 2004; Aaby \& Molbak, 1990), and therefore one major objective of the registry was to collect detailed maternal and infant data from birth. This included determining the twinning rate, with genetic distinction between monozygotic (MZ) and dizygotic (DZ) twins.

At the same time, we had an interest in twins from a metabolic perspective. Currently, diabetes rates are increasing rapidly across Sub-Saharan Africa (International Diabetes Federation, 2017). While a major part of this increase is driven by obesity, urbanization and longer life expectancy (Pastakia et al., 2017), a better understanding of other underlying factors is also needed. Previously, detrimental influences during fetal life have been suggested as an important risk factor for diabetes; for example, the 'thrifty phenotype hypothesis' (Hales \& Barker, 2001). Twins are particularly interesting in this regard, as their low birth weight could predispose them to diabetes later in life (Vaag \& Poulsen, 


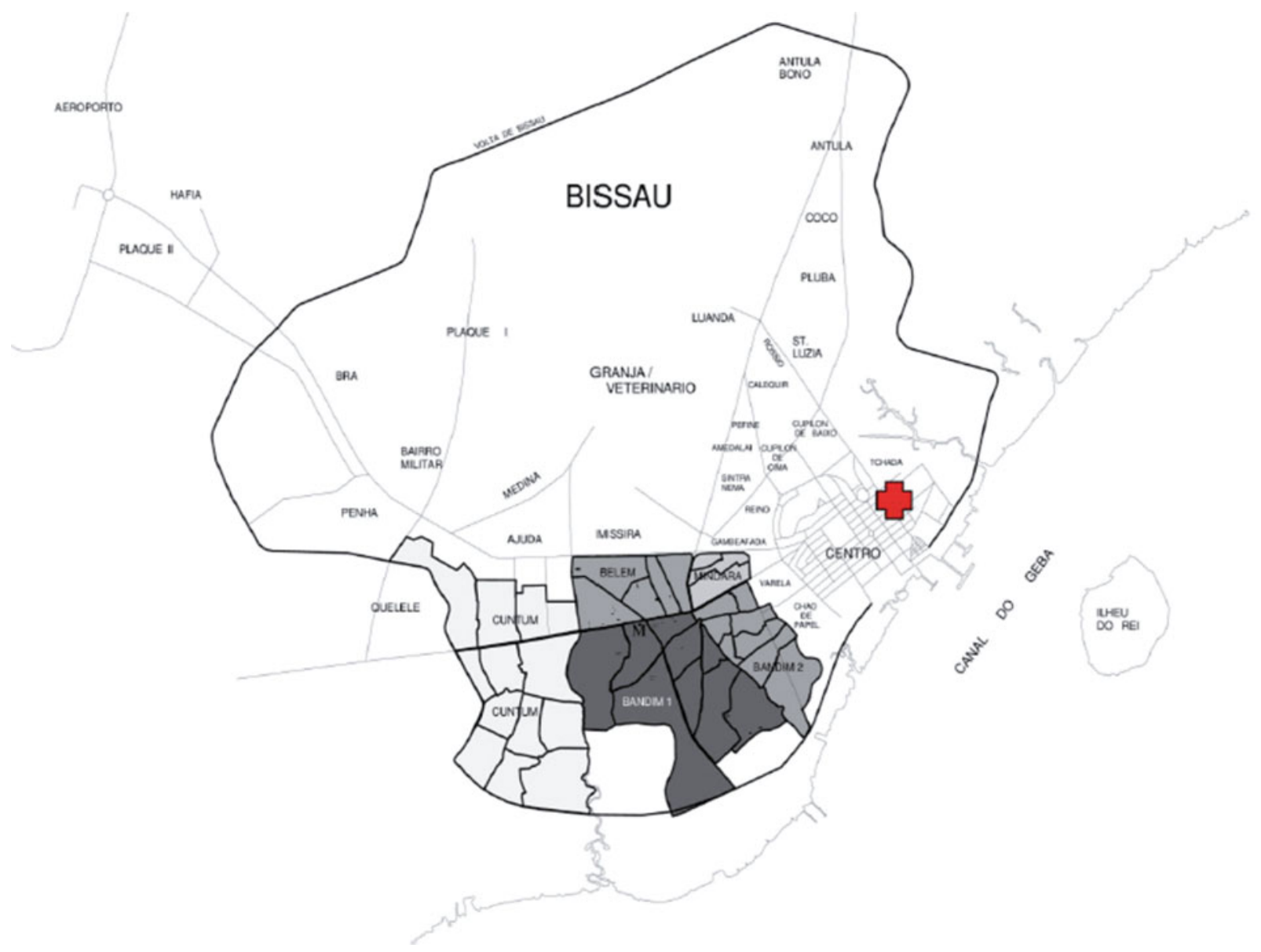

Fig. 1. Map of Bissau, Guinea-Bissau. The Bandim Health Project study area (HDSS) is marked in grey. The location of the HNSM is marked in red.

2007). Consequently, we wanted to determine whether young African twins had a higher rate of metabolic disorders (e.g., diabetes, metabolic syndrome [MS] and impaired glucose tolerance [IGT]) compared to singletons.

In this article, we provide an update on the Guinea-Bissau twin registry. The previous description of the registry in 2013 was published only a few years after the establishment and therefore largely focused on the design and structure of the cohort, and also on the overall objectives (Bjerregaard-Andersen et al., 2013a). The present report, however, contains a more detailed description of numbers enrolled and the current follow-up situation, as well as more of the main findings and future perspectives.

\section{Methods}

The registry was established in 2009 at the BHP (www.bandim.org) in Bissau, the capital of Guinea-Bissau. The BHP maintains an elaborate health and demographic surveillance system (HDSS) in six suburban areas of Bissau and has been doing so since 1978. Currently, the HDSS's population is approximately 100,000. All individuals within the HDSS are registered with unique ID numbers. Data are collected regarding movements in and out of the area, and births and deaths. Twin status (yes/no) is available. Children under 3 years of age are extensively followed by the HDSS, with repeated household visits every 3-4 months, and censuses of the whole population are carried out at 2-3-year intervals.
For several decades, the BHP has also maintained a presence at the Simão Mendes National Hospital (HNSM) in the center of Bissau (see Figure 1). The HNSM is the main referral hospital for the entire Guinea-Bissau. All births occurring at the hospital's maternity ward are thus registered by the BHP, as well as all hospitalizations at the adjacent pediatric ward. The hospital facilities are modest, and we have observed high overall mortality rates at both units (Biai et al., 2007; Bjerregaard-Andersen et al., 2018; Pinstrup Joergensen et al., 2018).

As a country, Guinea-Bissau is among the poorest in the world and has experienced long periods of political instability. This has also affected the health sector, which has been struggling to provide many basic services.

\section{Twin Registry}

The establishment and structure of the twin registry has previously been described (Bjerregaard-Andersen et al., 2013a). Briefly, two separate cohorts were created. The first was a cohort of newborn twins and was established at the HNSM hospital (Bjerregaard-Andersen et al., 2012). After maternal consent, newborn twins were prospectively enrolled on the day of birth or the day after, with detailed registration of pregnancy and birth-related data (including maternal HIV infection), weight and other anthropometry, clinical examination, possible malformations and maturity determined by Ballard score. Heel blood was collected for genetic analyses of zygosity in samesex pairs. After discharge, twins from the capital Bissau were driven 


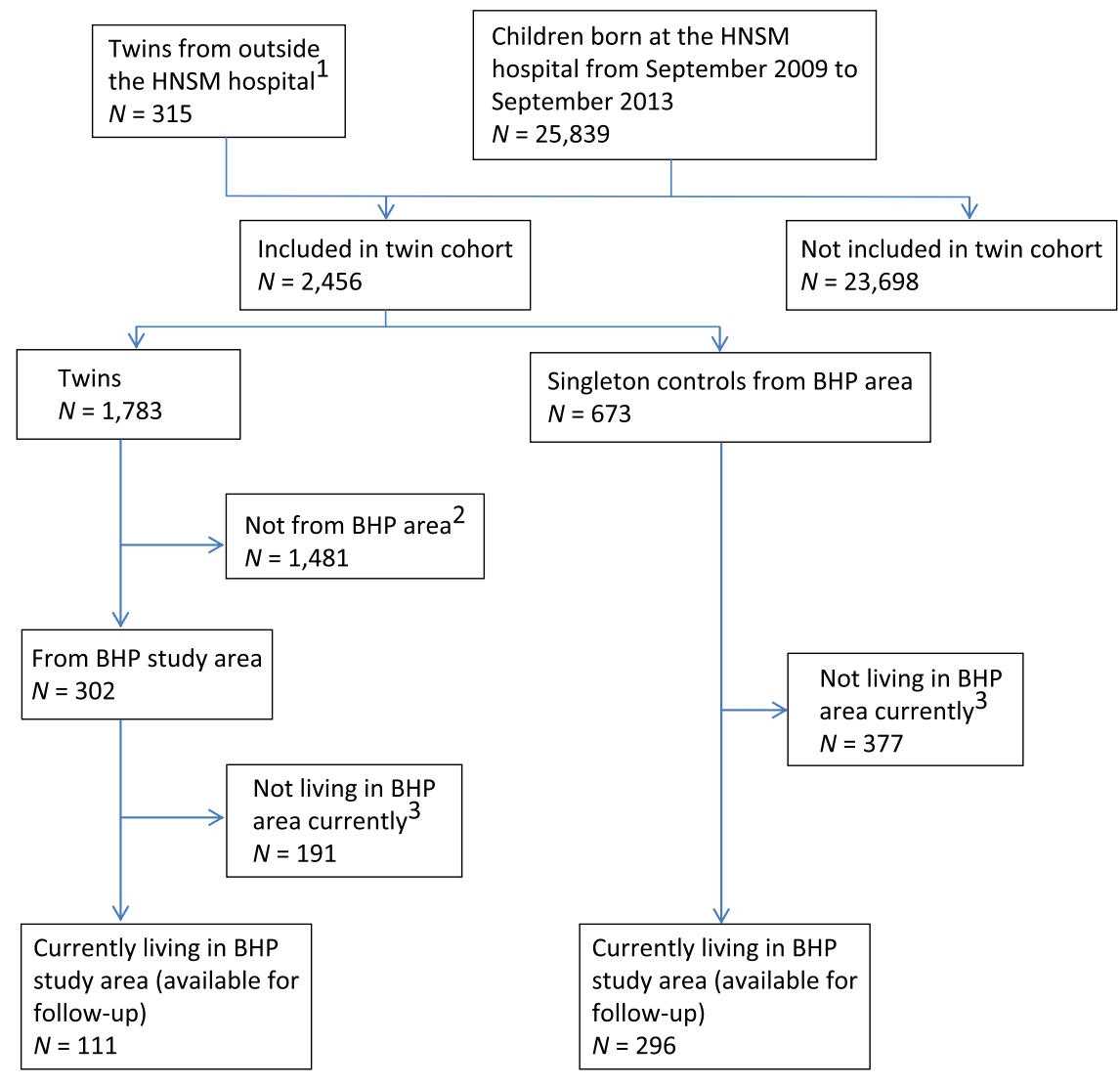

Fig. 2. Flowchart of newborn twin cohort - current followup situation.

Note: ${ }^{1} \mathrm{~A}$ number of twins were included at the Cumura Hospital outside Bissau or local health centers within Bissau. ${ }^{2}$ Comprise both the rest of the capital Bissau and those from rural areas. Those from the rest of Bissau were followed from 2009 to 2013 by the twin team. ${ }^{3}$ Includes migration, deaths and lost to follow-up. A number of mothers identified themselves as coming from the BHP area, though we were not able to find them in the HDSS database. home so that they could be followed by our twin team. Post-discharge follow-up was not possible outside Bissau. Follow-up took place at home after 2, 6 and 12 months, and afterwards at 6-month intervals (Bjerregaard-Andersen et al., 2014). At these visits, vital status was recorded, as well as anthropometric measurements, whether the mother was breastfeeding, hospital and health center consultations, and vaccinations. A control group of singleton deliveries among mothers from the BHP study area was created and followed as well.

Furthermore, we established a cohort of young twins aged between 5 and 30 years and living in the BHP study area (Bjerregaard-Andersen et al., 2013b). These twins were therefore all registered by the HDSS. The main objective of this cohort was to investigate metabolic disorders. Provided we obtained consent, the twins (or close family members) were interviewed regarding family disposition and symptoms indicative of diabetes. A physical examination was carried out, including blood pressure, height, weight, and waist and hip circumference. After an overnight fast, blood glucose was measured in the morning and blood samples were collected, processed and frozen for later biochemical analyses of hbalc, lipoproteins, c-peptide, insulin and B12/folic acid. Zygosity in same-sex pairs was evaluated by degree of physical similarity, but for a subset also by genetic testing of filter paper blood (similar to the newborn cohort). Birth weight was available from the HDSS, though often missing in the older records or if the person had been born at home. For all twins, an age-matched singleton control from the BHP study area was enrolled. In a substudy, a group of twins and singleton controls underwent oral glucose tolerance testing (OGTT) (Hennild et al., 2016). Here, we also estimated body fat percentage using skinfold measurements.

\section{Laboratory Measurements}

All advanced laboratory measurements have been carried out at Odense University Hospital, Denmark, as previously described, including genetic differentiation between $\mathrm{MZ}$ and $\mathrm{DZ}$ twins (Bjerregaard-Andersen et al., 2012), as well as the metabolic analyses (Bjerregaard-Andersen et al., 2013b). Additional biological material has been stored in a biobank.

\section{Ethics}

The investigations were approved by the Ethical Committee in Guinea-Bissau. Consultative approval was obtained from the Central Ethical Committee in Denmark. Written consent (either signature or fingerprint) was obtained in all cases. Future studies would, however, need new ethical approval.

\section{Discontinuation of Cohort of Newborn Twins}

Due to financial constraints, we were obliged to stop inclusions and follow-up in the cohort of newborn twins in September 2013. Follow-up of the twins coming from the BHP study area did, however, continue beyond that point through the HDSS and is still ongoing.

\section{Results}

The main results of the previous studies originating from the twin registry are briefly outlined here. 


\section{Cohort of Newborn Twins}

In the cohort of newborn twins, we recorded 1783 twins and 673 singleton controls from September 2009 to September 2013, at which point both inclusions and follow-up were discontinued for the majority of children. The follow-up did, however, continue for the newborn twins coming from the BHP study area (see Figure 2). The results of enrollments during the first 2 years (i.e., September 2009 to September 2011) have previously been reported (Bjerregaard-Andersen et al., 2012). We found very high perinatal twin mortality rates in both the overall community (218/ $1000)$ and at the hospital (237/1000). Compared to singletons, the perinatal twin mortality was much higher (relative risk $[R R]=2.7$, 95\% confidence interval (CI) $[1.9,3.8]$ ). The overall community twinning rate was 18 twin births per 1000 total births. MZ twinning occurred at a rate of 3.4/1000. Among twin mothers, $65 \%$ of those who delivered at the HNSM hospital were unaware of carrying twins throughout the pregnancy.

After the perinatal period (i.e., after the first 7 days of life), the infant twin mortality was significantly higher for twins compared to singletons, that is, $91 / 1000$ versus $42 / 1000$ person-years $(\mathrm{RR}=2.1,95 \%$ CI $[1.1,4.1]$; Bjerregaard-Andersen et al., 2014). The excess twin mortality was, however, concentrated during the first 3 months of life. Risk factors for twin death after the perinatal period (usually after discharge from the maternity ward) were birth weight $<2000 \mathrm{~g}(\mathrm{RR}=3.3,95 \% \mathrm{CI}[1.4,8.1])$, death of the cotwin perinatally $(\mathrm{RR}=2.5,95 \% \mathrm{CI}[1.2,5.6])$, severe maternal illness during pregnancy $(\mathrm{RR}=2.4,95 \% \mathrm{CI}[1.0,5.5])$ and maternal HIV infection ( $R R=3.2$, 95\% CI $[1.2,8.1])$. Verbal autopsies revealed that death occurred at home for the majority of both twins $(60 \%)$ and singletons (67\%). During infancy, hospitalization rates at the HNSM pediatric ward were similar for twins and singletons $(\mathrm{RR}=1.0,95 \% \mathrm{CI}[0.6,1.5])$. At 12 months after delivery, breastfeeding was significantly less common among HIV-infected twin mothers compared to uninfected, that is, $64 \%$ (7/11) versus $95.3 \%(122 / 128)$, respectively $(p<.001)$. Congenital malformations were much more often seen among twins, that is, $3.9 \%$ $(17 / 440)$ for twins versus $0.7 \%(2 / 301)$ for singletons $(R R=5.8$, 95\% CI $[1.3,25])$.

\section{Cohort of Young Twins}

Twins and singleton controls were enrolled in this cohort from October 2009 to August 2011 (Bjerregaard-Andersen et al., 2013b). In total, diabetes screening using hbalc was available for 574 twins and 463 singletons. The mean age for the two groups was 15.3 and 15.8 years, respectively $(p=.37)$. The mean birth weight (when available) was lower for twins than for singletons, 2.52 versus $3.10 \mathrm{~kg}$ (Diff $=0.58 \mathrm{~kg}$, 95\% CI [0.45, 0.71]). Mean $\mathrm{BMI}$ was also lower for twins, 17.7 versus $18.3 \mathrm{~kg} / \mathrm{m}^{2}(\mathrm{Diff}=0.60$, $95 \%$ CI $[0.011,1.19])$. The twin group included 187 twin pairs and 200 individual twins (i.e., only one twin was included). Genetic analysis of a subset of twin pairs revealed an MZ proportion of $18 \%$. By degree of physical similarity, the assistants were able to determine zygosity correctly in $89 \%$ of the same-sex twin pairs $(32 / 36)$.

We found no diabetes cases among the twins, while one young presumed type 1 diabetes case was identified among the singletons (fasting [F]-glucose $15.1 \mathrm{mmol} / \mathrm{L}$, hbalc $74 \mathrm{mmol} / \mathrm{mol}$. Insulin treatment was initiated). No difference was found in mean hbalc between twins and singletons, that is, $34 \mathrm{mmol} / \mathrm{mol}$ for both groups $(p=.33)$. Insulin resistance assessed by a homeostasis model (HOMA-IR) found no difference either $(p=.34)$. Using waist circumference, blood pressure, F-glucose and levels of triglycerides and HDL cholesterol to define the MS, we found an MS prevalence of 3.0\% (11/364) among twins and 3.6\% (13/360) among singletons $(p=.66)$. MS was more prevalent among twins if the co-twin had died $(p=.05)$.

A subset of the cohort (209 twins and 182 singleton controls) was investigated with OGTT from February 2011 to March 2012 (Hennild et al., 2016). Median age for those with an OGTT was 16.6 for twins and 14.2 years for singletons $(p=$ $.08)$. No difference was found in the prevalence of IGT between the two groups, that is, $2.5 \%(5 / 209)$ for twins and 3.5\% (6/182) for singletons $(p=.63)$. Twins did, however, have higher median glucose levels both in the fasting state ( 5.4 vs. $5.0 \mathrm{mmol} / \mathrm{L}, p=.001)$ and at $2 \mathrm{~h}$ after glucose intake (6.8 vs. $6.2 \mathrm{mmol} / \mathrm{L}, p=.001)$. A higher mean F-glucose level among twins, compared to singletons, was also seen in the main cohort (5.30 vs. $5.17 \mathrm{mmol} / \mathrm{L}, p=.01$ ) (Bjerregaard-Andersen et al., 2013b). Using calculations based on skinfold measurements, we found a higher median body fat percentage among twins than among singletons, $12.1 \%$ versus $11.1 \%(p=.02)$.

As this cohort consisted of young twins all from the BHP study area, it is being followed by the HDSS. As of the most recent census, 388 of twins ( $68 \%$ of the original sample) are currently living in the study area (see Figure 3).

\section{Discussion}

The Guinea-Bissau twin registry represents one of the first formal registries in Sub-Saharan Africa, the other being in Nigeria and focusing on cognitive functions (Hur et al., 2013). Several important findings have already been presented. Key to the GuineaBissau twin registry has been its close link to a well-established HDSS at the BHP, not only enabling us to retrieve previously collected information, but also conducting future studies as the twins can be visited again.

\section{Consistency with Other Findings}

In the cohort of newborn twins, we identified a twinning rate (18/ 1000) comparable to neighboring West African countries (Bjerregaard-Andersen et al., 2012). As opposed to DZ twinning, the MZ twinning rate is considered fairly constant globally at around 3.5-4/1000 (Pison, 1992), which is close to our finding. The perinatal twin mortality rate was, however, disturbingly high at around 22\% (Bjerregaard-Andersen et al., 2012). This reflects the limited health services available to pregnant women in Guinea-Bissau, which was underscored by the fact that most mothers of twins did not know they were carrying twins. We have previously found very high stillbirth rates in the general population (Bjerregaard-Andersen et al., 2018), but even in the absence of ultrasound, a thorough clinical examination alone can detect the majority of twin pregnancies (Groot \& Buchmann, 2003). Thus, twin pregnancies correctly recognized prior to delivery can be used as a proxy performance indicator of antenatal care in low income settings such as Bissau (Bjerregaard-Andersen et al., 2012).

Twin mortality remained elevated during the first months of life. In some cases, it is likely that discharge from the maternity ward took place too early (Bjerregaard-Andersen et al., 2014). A recent study from neighboring Gambia found that excess twin mortality continued until the end of infancy (Miyahara et al., 2016). In our data, one important observation was the strong influence of maternal health on survival of twins, particularly with regard to maternal HIV infection. As antiretroviral treatment 


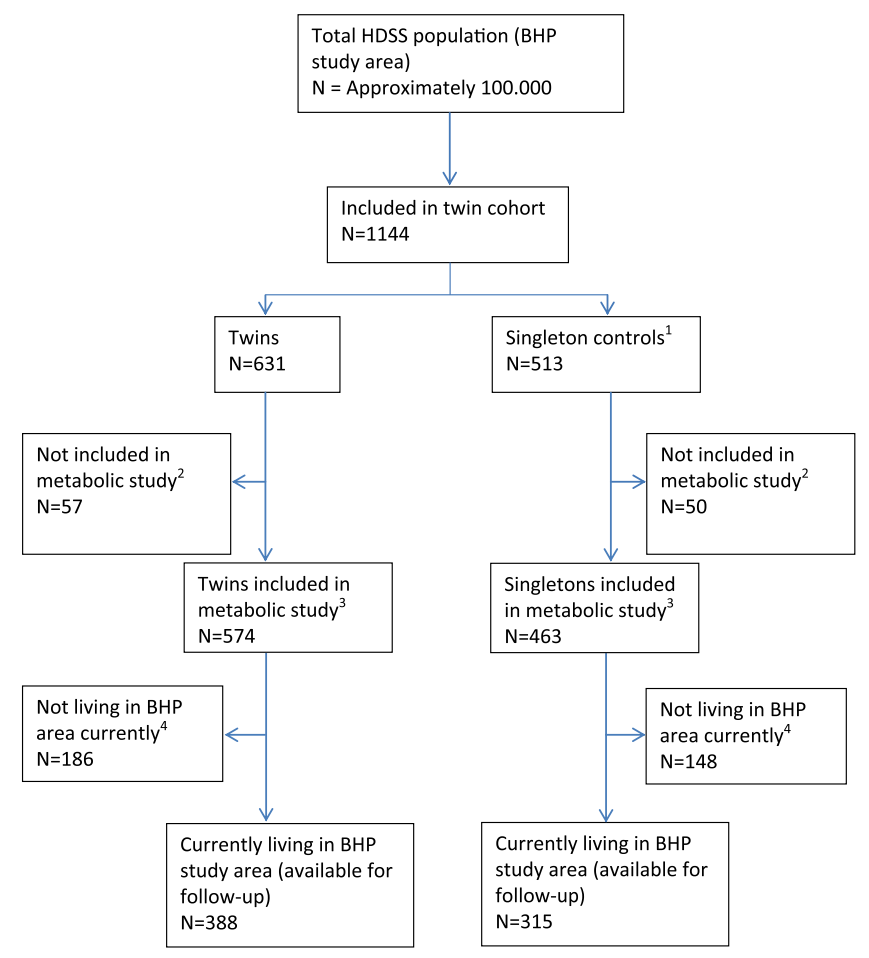

Fig. 3. Flowchart of cohort of young twins - current follow-up situation. (1) Matched on age. (2) Due to refusal to give blood or not enough blood for analysis. (3) 209 of the twins and 182 of the singleton controls were included in OGTT sub-study. Biobank material is stored. (4) Includes migration, deaths and lost to follow-up.

was usually administered during delivery among HIV-infected mothers in order to prevent mother-to-child transmission, many of the twins may not have been HIV infected themselves. A study from Zambia found a high infant mortality among children of HIV-infected mothers, even when these children were not HIV infected (Kuhn et al., 2005). The authors identified several possible reasons, including maternal co-infection with opportunistic pathogens, reduced transplacental transfer of maternal antibodies, poorer breast milk and lack of breastfeeding. HIV-infected mothers may also be too sick or have too little energy to properly care for their children, a problem likely exacerbated in the case of twin offspring.

Notably, despite being a high-risk group, many twin deaths occurred outside hospital, and twins did not have higher hospitalization rates than singletons (Bjerregaard-Andersen et al., 2014). Previously, it has been suggested that there may be obstacles related to twin hospital admissions in emergency situations, including financial constraints in twin families (Justesen \& Kunst, 2000).

Due to their high mortality and the fact that twinning is frequent in Africa, it has been estimated that twins account for $15.1 \%$ of neonatal mortality and $10.7 \%$ of the child mortality in the region (Monden \& Smits, 2017). In the Gambian twin study, it was observed that although the general neonatal and infant mortality had dropped considerably over the years, a similar decline was not observed for twins (Miyahara et al., 2016). Thus, addressing twin mortality during early life should be a priority. Previously, we have outlined how such interventions would include improved antenatal care (particularly with a focus on recognizing twin pregnancies), that twin deliveries take place in hospitals adequately equipped and staffed to perform emergency cesarean sections and neonatal resuscitation, and that twins are carefully evaluated before hospital discharge (Christensen \& Bjerregaard-Andersen,
2017). Strategies recommended for a reduction of stillbirths in the general population (e.g., proper fetal monitoring during labor, use of partograms and perinatal audits; Bjerregaard-Andersen et al., 2018) would likely also benefit twins considerably. Ideally, twins should also receive home visits during infancy, with a focus on nutrition and failure to thrive. Here, twins born from HIVinfected mothers represent a particular high-risk group.

Despite twins in Guinea-Bissau having a much lower birth weight compared to singletons, we did not find higher rates of diabetes, MS and IGT among young twins in our studies (BjerregaardAndersen et al., 2013b; Hennild et al., 2016). This is in line with large-scale register studies from Scandinavia (Petersen et al., 2011). In fact, based on glucose metabolism among extremely birth weight discordant $\mathrm{MZ}$ twin pairs, it has been proposed that there are common genetic factors in the relationship between low birth weight and adult metabolic disease (Frost et al., 2012). Interestingly, twins in our cohort did, however, have higher median glucose levels in both the fasting and in the post-prandial state, and also a higher body fat percentage (Hennild et al., 2016). As our cohort was young, we cannot exclude that our findings could be early indicators of an increased diabetes risk later in life. Elsewhere, several clinical studies have found an association between twin status and dysglycaemia (Poulsen et al., 2009; Vaag \& Poulsen, 2007), particularly among MZ twins (Poulsen \& Vaag, 2006).

To our knowledge, apart from the investigations from GuineaBissau, no other metabolic twin studies are available from Sub-Saharan Africa. It should also be noted that findings from high-income countries may not be directly applicable to African twins. Different types of dietary habits can affect fetal conditions in utero, as can common infections such as malaria and HIV. Furthermore, due to the very high early-life mortality, one 
could consider whether African twins who survive into adulthood by nature are quite strong individuals and thereby somewhat protected against dysglycaemia, that is, a 'healthy survivor bias.' On the other hand, it could also be argued that the hard conditions (e.g., insufficient nutrition) experienced both in utero and in early life would make African twins even more vulnerable.

\section{Perspectives}

Based on the collected data, there is an urgent need to improve twin health care in Guinea-Bissau. Most importantly, local health care professionals need to be aware that newborn twins constitute a high-risk group, and that a reduction in twin mortality is important in lowering the overall number of childhood deaths. Though enrollment and follow-up have been discontinued in a large part of the newborn cohort (i.e., those not followed by our HDSS), the Guinea-Bissau twin registry has obtained valuable mortality data. Future analyses are thus possible, including on the effects of 'natural crowding' and infectious disease transmission in twin pairs, which have previously been studied at the BHP (Aaby \& Molbak, 1990).

Diabetes is already a considerable problem in Bissau, albeit mainly above 35 years of age (Carvalho et al., 2018). Though known driving factors such as obesity, sedentary lifestyle and urbanization likely play a major role, detrimental influences during fetal life may also be important in the pathogenesis (Tomar et al., 2015). Here, the Guinea-Bissau twin registry could be of continued use in future metabolic research. One obvious study would be to repeat the metabolic investigations once all the twins have reached adulthood.

The most important aspect of this work has, however, been the creation of the registry itself, as it offers a unique platform for studying life course health among twins in an African population. Though we have previously focused on metabolic disorders, the registry could in the future also be used in studying variations in other types of disease (e.g., cardiovascular and infectious) by applying the various types of twin study designs. In this context, it should be noted that while research funding in Sub-Saharan Africa is often allocated to studies with relatively short time frames, longitudinal cohorts such as the Guinea-Bissau twin registry open new insights into disease development in low-income settings. Maintaining such cohorts (potentially spanning over decades) is therefore important, a fact which should be recognized by funding agencies.

\section{Conclusion}

Over the last 10 years, the Guinea-Bissau twin registry has contributed important findings from a low-income African population. Our studies have focused on both the very high mortality among twins during early life and the possible long-term metabolic risks associated with fetal undernutrition. As the registry is based on an elaborate HDSS following a large part of the population in the capital Bissau, future twin studies are possible.

Acknowledgments. The authors would like to thank all the participating twins and singleton controls, as well as their mothers, for their important contribution. The authors would also like to thank the twin team at the Bandim Health Project in Guinea-Bissau; in particular, Maria Rosa Fernandes, Fiel Gomes, Delmiro Gomes Barbosa, Moises Soares, Leontina Indeque, Lita Indeque, Luis Camala and Abdalaha Umaro Cande. In terms of laboratory analyses, the authors would like to thank Lone Hansen, Steno Diabetes Center Odense and Lene Christiansen, Department of Clinical Immunology, Rigshospitalet, Denmark. The authors would also like to thank Prof. Christine Stabell Benn (CVIVA) and Amabelia Rodrigues (BHP) for valuable inputs. Special thanks go to Dr Luis Carlos Joaquím from the Guinean Diabetes Association who took part in this work from the beginning, but sadly died from illness in 2012.
Conflicts of interest. None.

\section{References}

Aaby, P., \& Molbak, K. (1990). Siblings of opposite sex as a risk factor for child mortality. British Medical Journal, 301, 143-145.

Aaby, P., Jensen, H., Rodrigues, A., Garly, M. L., Benn, C. S., Lisse, I. M., \& Simondon, F. (2004). Divergent female-male mortality ratios associated with different routine vaccinations among female-male twin pairs. International Journal of Epidemiology, 33, 367-373.

Akinboro, A., Azeez, M. A., \& Bakare, A. A. (2008). Frequency of twinning in southwest Nigeria. Indian Journal of Human Genetics, 14, 41-47.

Biai, S., Rodrigues, A., Gomes, M., Ribeiro, I., Sodemann, M., Alves, F., \& Aaby, P. (2007). Reduced in-hospital mortality after improved management of children under 5 years admitted to hospital with malaria: Randomised trial. British Medical Journal, 335, 862-862.

Bjerregaard-Andersen, M., Biering-Sorensen, S., Gomes, G. M., Bidonga, A., Jensen, D. M., Rodrigues, A., .. Sodemann, M. (2014). Infant twin mortality and hospitalisations after the perinatal period - A prospective cohort study from Guinea-Bissau. Tropical Medicine \& International Health, 19, 1477-1487.

Bjerregaard-Andersen, M., Gomes, M. A., Joaquim, L. C., Rodrigues, A., Jensen, D. M., Christensen, K., ... Sodemann, M. (2013a). Establishing a twin registry in Guinea-Bissau. Twin Research and Human Genetics, 16, 179-184.

Bjerregaard-Andersen, M., Hansen, L., da Silva, L. I., Joaquim, L. C., Hennild, D. E., Christiansen, L., .. Beck-Nielsen, H. (2013b). Risk of metabolic syndrome and diabetes among young twins and singletons in Guinea-Bissau. Diabetes Care, 36, 3549-3556.

Bjerregaard-Andersen, M., Lund, N., Jepsen, F. S., Camala, L., Gomes, M. A., Christensen, K., . . Sodemann, M. (2012). A prospective study of twinning and perinatal mortality in urban Guinea-Bissau. BMC Pregnancy Childbirth, $12,140$.

Bjerregaard-Andersen, M., Lund, N., Pinstrup Joergensen, A. S., Starup Jepsen, F., Werner Unger, H., Mane, M., .. S Stabell Benn, C. (2018). Stillbirths in urban Guinea-Bissau: A hospital- and community-based study. PLoS One, 13, e0197680.

Carvalho ACC., Naforna N., \& Santos G. (2018). A Prevalência da Diabetes Mellitus e Obesidade na População Adulta da Guiné-Bissau: Um Estudo Piloto. Revista Portuguesa de Endocrinologia, Diabetes e Metabolismo, 13, 2-6.

Christensen, K., \& Bjerregaard-Andersen, M. (2017). Twin-singleton earlylife survival in sub-Saharan Africa. Lancet Global Health, 5, e636-e637.

Frost, M., Petersen, I., Brixen, K., Beck-Nielsen, H., Holst, J. J., Christiansen, L., .. Christensen, K. (2012). Adult glucose metabolism in extremely birthweight-discordant monozygotic twins. Diabetologia, 55, 3204-3212.

Groot, M. C., \& Buchmann, E. J. (2003). Where there is no ultrasound: Delay and failure of antenatal diagnosis of twin pregnancy. Tropical Doctor, 33, 5-7.

Hales, C. N., \& Barker, D. J. (2001). The thrifty phenotype hypothesis. British Medical Bulletin, 60, 5-20.

Hennild, D. E., Bjerregaard-Andersen, M., Joaquim, L. C., Christensen, K., Sodemann, M., Beck-Nielsen, H., \& Jensen, D. M. (2016). Prevalence of impaired glucose tolerance and other types of dysglycaemia among young twins and singletons in Guinea-Bissau. BMC Endocrine Disorders, 16, 46.

Hur, Y. M., Kim, J. W., Chung, K. W., Shin, J. S., Jeong, H. U., \& Auta, E. (2013). The Nigerian twin and sibling registry. Twin Research and Human Genetics, 16, 282-284.

International Diabetes Federation. (2017). IDF diabetes atlas. https://www.idf.org/ Justesen, A., \& Kunst, A. (2000). Postneonatal and child mortality among twins in Southern and Eastern Africa. International Journal of Epidemiology, 29, 678-683.

Kuhn, L., Kasonde, P., Sinkala, M., Kankasa, C., Semrau, K., Scott, N., ... Thea, D. M. (2005). Does severity of HIV disease in HIV-infected mothers affect mortality and morbidity among their uninfected infants? Clinical Infectious Diseases, 41, 1654-1661.

Miyahara, R., Jasseh, M., Mackenzie, G. A., Bottomley, C., Hossain, M. J., Greenwood, B. M., ... Roca, A. (2016). The large contribution of twins to neonatal and post-neonatal mortality in The Gambia, a 5-year prospective study. BMC Pediatrics, 16, 39. 
Monden, C. W. S., \& Smits, J. (2017). Mortality among twins and singletons in sub-Saharan Africa between 1995 and 2014: A pooled analysis of data from 90 Demographic and Health Surveys in 30 countries. Lancet Global Health, 5, e673-e679.

Pastakia, S. D., Pekny, C. R., Manyara, S. M., \& Fischer, L. (2017). Diabetes in sub-Saharan Africa - From policy to practice to progress: Targeting the existing gaps for future care for diabetes. Diabetes, Metabolic Syndrome and Obesity, 10, 247-263.

Petersen, I., Nielsen, M. M., Beck-Nielsen, H., \& Christensen, K. (2011). No evidence of a higher 10 year period prevalence of diabetes among 77,885 twins compared with 215,264 singletons from the Danish birth cohorts 1910-1989. Diabetologia, 54, 2016-2024.

Pinstrup Joergensen, A. S., Bjerregaard-Andersen, M., Biering-Sorensen, S., Byberg, S., Camala, L., Martins, C., ... Stabell Benn, C. (2018). Admission and mortality at the main neonatal intensive care unit in Guinea-Bissau. Transactions of the Royal Society of Tropical Medicine and Hygiene, 112, 335-341.
Pison, G. (1992). Twins in Sub-Saharan Africa: Frequency, social status and mortality. In E. van de Walle, G. Pison, \& M. Sala-Diakanda (Eds.), Mortality and society in Africa (pp. 253-278). Oxford, UK: Clarendon Press.

Poulsen, P., \& Vaag, A. (2006). The intrauterine environment as reflected by birth size and twin and zygosity status influences insulin action and intracellular glucose metabolism in an age- or time-dependent manner. Diabetes, 55, 1819-1825.

Poulsen, P., Grunnet, L. G., Pilgaard, K., Storgaard, H., Alibegovic, A., Sonne, M. P., ... Vaag, A. (2009). Increased risk of type 2 diabetes in elderly twins. Diabetes, 58, 1350-1355.

Tomar, A. S., Tallapragada, D. S., Nongmaithem, S. S., Shrestha, S., Yajnik, C. S., \& Chandak, G. R. (2015). Intrauterine programming of diabetes and adiposity. Current Obesity Reports, 4, 418-428.

Vaag, A., \& Poulsen, P. (2007). Twins in metabolic and diabetes research: What do they tell us? Current Opinion in Clinical Nutrition and Metabolic Care, 10, 591-596. 